\title{
Kernos
}

Revue internationale et pluridisciplinaire de religion grecque antique

18 | 2005

Varia

\section{Louis GERNET, Polyvalence des images. Testi e frammenti sulla leggenda greca}

\section{Vinciane Pirenne-Delforge}

\section{OpenEdition \\ Journals}

\section{Édition électronique}

URL : http://journals.openedition.org/kernos/1748

DOI : 10.4000/kernos. 1748

ISSN : 2034-7871

\section{Éditeur}

Centre international d'étude de la religion grecque antique

\section{Édition imprimée}

Date de publication : 1 janvier 2005

Pagination : $562-564$

ISSN : 0776-3824

\section{Référence électronique}

Vinciane Pirenne-Delforge, "Louis gernet, Polyvalence des images. Testi e frammenti sulla leggenda greca », Kernos [En ligne], 18 | 2005, mis en ligne le 24 mai 2011, consulté le 21 septembre 2020. URL http://journals.openedition.org/kernos/1748 ; DOI : https://doi.org/10.4000/kernos.1748 
Dans de telles conditions, il serait en tout cas nécessaire de pourvoir à nouveau le terme « orphique » de guillemets, de l'intitulé de l'ouvrage jusqu'à la dernière ligne du commentaire ( $c f$. p. 128); et il serait certainement indispensable de recourir en parallèle à l'ouvrage de A. Bernabé \& A.I. Jiménez San Cristóbal, Instrucciones para el más allá. Las laminillas órficas de oro, Madrid, 2001 (qui présente un commentaire circonstancié des aspects les plus importants offerts par toutes les lamelles d'or lamelles de Pella incluses $=$ fr. $496 \mathrm{~F}$ Bernabé, - présentées en traduction et accompagnées de l'édition des textes correspondants avec un apparat exhaustif et une bibliographie complète, ainsi que d'un dossier iconographique). N'eût-il d'ailleurs pas été plus opportun d'offrir en traduction au public francophone ce dernier ouvrage?

Claude Calame

(EHESS, Paris)

Agnès Pigler, Plotin. Traité 54 (I, 7), Introduction, traduction, commentaires et notes, Paris, Éd. du Cerf, 2004, 1 vol. 12,5×19,5 cm, 194 p. (coll. Les écrits de Plotin). ISBN : 2-204-07415-2.

Cette prestigieuse collection, entreprise à l'initiative et sous la direction de Pierre Hadot, à qui l'on doit la publication des trois premiers volumes (cf. Kernos 1 [1988], p. 253; 5 [1992], p. 349; 8 [1995], p. 307-308) progresse à pas lents mais assurés. Le présent traité, qui est le huitième paru, est le dernier qu'ait écrit Plotin. Très bref - sa traduction compte à peine huit pages -, mais d'une grande densité, il traite "Du premier Bien et des autres biens » et peut être vu comme une petite somme de l'enseignement du philosphe, une sorte de testament spirituel. Plotin y fait sienne la doctrine platonicienne d'un Bien transcendant, ce qui ne l'empêche pas d'adhérer aussi à la conception aristotéliciene d'un Premier moteur immobile. Pour se rapprocher de ce Bien absolu, l'âme est invitée à conformer sa vie à celle de l'intelligence, mais c'est lorsqu'elle est séparée du corps par la mort qu'elle parvient à s'unir plus pleinement au Bien. Cette méditation éthique et métaphysique comporte assurément une dimension religieuse, mais les références à la religion traditionnelle y sont rares. On y parle une fois de l'Hadès comme lieu de la sanction d'une vie mauvaise et, une fois aussi, il y est aussi question des dieux - en l'occurrence les astres - qui « possèdent le Bien sans aucun mal».

A.P., qui enseigne la philosophie à l'Université de Dijon, donne de ce texte une traduction rigoureuse. Son introduction et ses commentaires, fort abondants mais dépouillés de toute vaine érudition, témoignent d'une bonne connaissance de l'œuvre de Plotin et s'avèrent très éclairants pour le lecteur. Comme de coutume, une bibliographie et plusieurs index complètent le travail. Cette publication répond donc bien aux objectifs initiaux qu'avait définis le directeur de la collection et suit fidèlement les modèles qu'il a lui-même donnés.

André Motte (Université de Liège)

Louis GERnet, Polyvalence des images. Testi e frammenti sulla leggenda greca, editi da Antonella Soldani, contributi di Michela Benedetti, Vanessa Ghionzoli, Lucia Marrucci, andrea Taddei, prefazione di Riccardo Di Donato, Pisa, Edizioni ETS, 2004. 1 vol. 15,5 $\times 22$ cm, 287 p. (Antropoi. Studi e materiali di Antropologia storica del mondo antico, 1). ISBN : 88-467-0945-4. 
L'historiographie en tant que regard d'une discipline sur elle-même a récemment ouvert quelques beaux chantiers. L'édition de correspondances entre savants européens en est une manifestation particulièrement féconde et significative. L'entrée dans le laboratoire des chercheurs par une étude rigoureuse de leurs archives en est une autre. Avec ce livre, Riccardo di Donato et de jeunes chercheurs de Pise ajoutent, depuis l'Italie, une pierre à l'édifice de cette historiographie. Dépositaire des « archives Louis Gernet », l'équipe en livre ici une sélection cohérente, articulée autour de "la légende grecque ». Cette incursion dans le laboratoire d'un intellectuel aussi indépendant que Gernet, qui a eu une influence importante sur Vernant et Detienne, pour ne citer qu'eux, est un voyage passionnant dans la genèse « d'un essai général d'analyse du mythe pour l'intelligence de la préhistoire sociale » (lettre à I. Meyerson du 8 juillet 1942), un essai qui sera fragmenté dans des contributions singulières (dont une des plus marquantes est sans conteste La notion mythique de la valeur en Grèce [Journal de psychologie 1948, repris dans Anthropologie de la Grèce antique, 1968, p. 93-137]).

Cet ouvrage soigneux satisfait aux exigences scientifiques d'un travail archivistique et se voit accompagné d'un apparat critique précis. Il est divisé en trois parties, chacune introduite par un long commentaire de forme et de fond, qui met également en perspective certaines archives non publiées. Le premier groupe, affecté par Gernet lui-même de la lettre $\psi$, relevait de la «psychologie », du "travail mental ». C'est là que se rencontre l'expression «polyvalence des images»- qui a donné son titre au livre. Elle est définie par Gernet comme «un phénomène de mémoire sociale » dans la mesure où des représentations (des schèmes) « ont correspondu à des objets divers de préoccupation ou d'intérêt dans des milieux successifs » (p. 48) : c'est véritablement le «fil rouge» de la réflexion portée par les brouillons qui sont ici présentés. Une analyse assez longue de la «légende de Bellérophon » montre à la fois l'originalité de Gernet (la mise en perspective 'structurale' des épreuves du héros) et l'obligation de se poser des questions de l'époque, même s'il ne les considérait pas comme essentielles (origine de la légende). Forcément, bon nombre de thèmes ne sont qu'ébauchés et d'autres sont repris et retravaillés dans les trois parties (Thésée, Érysichthon). La deuxième partie ( $Г \varrho$ 'Grèce') regroupe tout une série de réflexions autour de noyaux thématiques: la mort consacrante de jeunes héros, aspects de la légende de Thésée, Ino, Jason en Colchide et le labourage sacré, l'agneau d'or des Atrides - et autres talismans dorés -, l'eranos et Polydecte/Persée, Érysichthon. La troisième partie semble avoir été conçue par son auteur comme une «conclusion» (Schluss) qui renvoie notamment à un "titre » et à un " chapitre d'introduction» (p. 202). Cette conclusion montre de façon lumineuse la méthode de travail de Gernet et ses principes d'analyse. Le questionnement sur la légende pose une problématique d'histoire - en l'occurrence l'information qu'elle recèle sur le passé de la société où elle s'est constituée. Mais Gernet précise d'emblée qu'il ne s'agit pas à proprement parler de « rechercher dans la légende des éléments prétendus historiques» (par ex. le fait que tel héros ait ou non existé) : "nous nous attacherons de préférence à ce qu'il y a de mythique au sens courant... dans la légende » lorsque la distance n'est pas trop grande « entre le thème mythique et l'histoire humaine qui en est sortie» (p. 192). C'est ainsi qu'il donne à la figure d'Hélène le statut de «curiosité » qui marque l'inclinaison de la légende vers le roman et cesse dès lors d'être instructive. Dans une réflexion d'aujourd'hui, cette figure ne recèlerait pas moins que d'autres, comme Thésée ou Bellérophon, une dimension proprement mythique, si l'on ne réduit pas le «mythique » à l'évocation d'une pratique sociale. Gernet en semble conscient par ailleurs et s'oppose à la «théorie du reflet». La légende n'est pas qu'une illustration et la mythologie «correspond à une fonction mentale autonome, qui n'est autre que la fonction esthétique, c'est-à-dire celle de la 
pensée par images. Il faut donc considérer le jeu des images pour lui-même... on en sera plus sûrement récompensé qu'à faire la chasse aux 'usages primitifs', maigre butin épars'»(p. 202). C'est donc le travail de la légende sur les représentations et les pratiques d'une société qu'il analyse, dans un cheminement d'une étonnante modernité. L'ouvrage se referme sur un inventaire des archives, une bibliographie et un index des noms antiques.

Vinciane Pirenne-Delforge (FNRS - Université de Liège)

\section{Actes de colloques, ouvrages collectifs et mélanges}

AcCorinti Domenico, Chuvin Pierre (éds), Des Géants à Dionysos. Mélanges de mythologie et de poésie grecque offerts à Francis Vian, Alessandria, Ed. dell'Orso, 2003 (Hellenica. Testi e strumenti di letteratura greca antica, medievale e umanistica, 10).

D. ACCORINTI, Parturiunt montes an parturiuntur? La nascita delle montagne nel mito, p. 124; A. Bernabé, Autour du mythe orphique sur Dionysos et les Titans. Quelques notes critiques, p. 25-39; A. CAMERON, A Greek source of Ovid's Metamorphoses?, p. 41-59; J. YOYOTTE, P. CHUVIN, Autour du solstice d'hiver: Épiphane et les fêtes alexandrines de l'éternité, p. 135-145; W. FAUTH, Kosmische Katastrophen im griechischen Mythos, p. 61-73; Ch. PIETSCH, Einheit oder bunte Fülle? Zu Funktion und Integration des Mythos in den Epinikien des Bakchylides am Beispiel des Herakles-Meleager-Mythos in B. 5, p. 173-188; A. KÖHNKEN, Apoll-Aitien bei Kallimachos und Apollonios, p. 207-213; P. CHuvin, Anaphé, ou la dernière épreuve des Argonautes, p. 215-221; S. SAÏD, Divination et devins dans les Argonautiques, p. 255-275; J.L. LigHTFOOT, Giants and Titans in Oracula Sibyllina 1-2, p. 393-401; H. FrangOUlis, Les pierres magiques dans les Dionysiaques de Nonnos de Pannopolis, p. 433-445.

Alvar EzQuerra Antonio, González Castro José Francisco (éds.), Actas del XI Congreso Español de Estudios Clásicos (Santiago de Compostela, del 15 al 20 de septiembre de 2003), Madrid, 2005, vol. I.

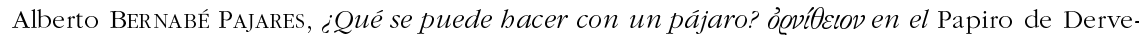
ni, p. 287-297; Francesc CASADESÚs BORDOY, Adaptaciones e interpretaciones estoicas de los poemas de Orfeo, p. 309-18; Ana Isabel JimÉNEZ SAN CRISTÓBAL, El concepto de dike en el orfismo, p. 351-361; Raquel MARTín HERnÁNDEZ, Orfeo, el orfismo y la magia en los s. Vy IV a. C., p. $375-$ 383; Inmaculada Rodríguez Moreno, Influencias de los Oráculos Caldeos en Porfirio de Tiro,

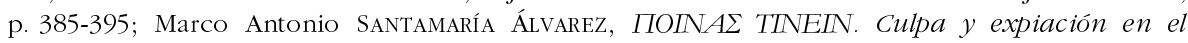
orfismo, p. 397-405; Pilar DÍEZ DEL CORRAL CORREDOIRA, Los contextos del amor. Eros en las imágenes de cortejo de Dioniso y Ariadna, p. 431-441; Fátima DíEz Platas, Agua para los vivos, agua para los muertos: sobre iconografía y función en los lutróforos áticos de época arcaica, p. 443-453; Jesús-María NiETO IBÁÑEZ, El mito de Tifón y el antijudaísmo de Egipto, p. 525-534; María Elena Rodríguez Ten, Las Dédalas de Platea: ¿un festival de Hera?, p. 569-577.

Ambos Claus, Hotz Stephan, Schwedier Gerald, Weinfurter Stefan (éds), Die Welt der Rituale. Von der Antike bis heute, Darmstadt, Wissenschaftliche Buchgesellschaft, 2005.

E. Stavrianopoulou, Die „gefahrvolle“ Bestattung von Gambreion, p. 24-37; ead., Priester gesucht, Erfahrung erwünscht!, p. 90-95; St. HOTZ, Delphi-ein störrische Ziege und Priester unter Druck, p. 102-105; A. CHANIOTIS, Ein mißverstandenes Ritual der griechischen Diplomatie: Geschichte als Argument, p. 106-109; St. HOTZ, Eine „grenzüberschreitende“ Prozession in Athen, p. 133-136; E. Stavrianopoulou, Göttin Bendis in Attika, p. 144-155; A. Chaniotis, Akzeptanz 